\title{
Deterministic qubit transfer between orbital and spin angular momentum of single photons
}

\author{
Vincenzo D’Ambrosio ${ }^{1}$, Eleonora Nagali ${ }^{1}$, Carlos H. Monken ${ }^{2}$, Sergei Slussarenko ${ }^{3}$, Lorenzo Marrucci ${ }^{3}$, Fabio \\ Sciarrino $^{1,4, *}$ \\ ${ }^{1}$ Dipartimento di Fisica, Sapienza Università di Roma, Roma 00185, Italy \\ ${ }^{2}$ Departamento de Fisica, Universidade Federal de Minas Gerais, Caixa Postal 702, Belo Horizonte, MG 30161-970, Brazil \\ ${ }^{3}$ Dipartimento di Scienze Fisiche, Università di Napoli "Federico II", Compl. Univ. di Monte S. Angelo, 80126 Napoli, Italy \\ ${ }^{4}$ Istituto Nazionale di Ottica Applicata, Firenze, Italy \\ *Corresponding author: fabio.sciarrino@uniroma1.it
}

Compiled October 3, 2011

In this work we experimentally implement a deterministic transfer of a generic qubit initially encoded in the orbital angular momentum of a single photon to its polarization. Such transfer of quantum information, completely reversible, has been implemented adopting a electrically tunable q-plate device and a Sagnac interferomenter with a Dove's prism. The adopted scheme exhibits a high fidelity and low losses. (c) 2011 Optical Society of America

OCIS codes: (270.0270) Quantum Optics, (270.5585) Quantum information and processing

Qubits are often encoded in the polarization state of photons. This is essentially due to the ease of manipulation and detection of the spin angular momentum (SAM) of light. Besides SAM, photons can carry orbital angular momentum (OAM) [1], which is related to the spatial distribution of the field. Photon states with a well defined orbital angular momentum are the ones characterized by an azimuthal dependence $e^{i l \phi}$ of the phase front, where $l$ is an integer (e.g. Laguerre-Gauss modes) [2]. The Hilbert space associated with the OAM degree of freedom is infinite-dimensional, while the one associated with polarization is restricted to two dimensions. This fact suggests the use of OAM, alone or coupled with spin, as a resource to encode information in higher dimensional quantum states, or qudits [3-5]. A number of devices have been developed for the generation and manipulation of OAM photon eigenstates, including holograms [2, 6], mode converters [7], spiral phase plates [8], and more recently the liquid-crystal q-plate (QP) [9]. The latter device, in particular, introduces a controlled coupling between spin and orbital angular momentum of a single photon, allowing for a coherent transfer of information between the spaces associated with these two degrees of freedom [10]. This feature has been recently exploited for implementing a probabilistic quantum transferrer, i.e. a device that can transfer a qubit from a degree of freedom to another and vice versa with a theoretical success probability of 50\% [11]. The transfer has been demonstrated in particular from the bidimensional space of polarization $\pi$ to a bidimensional subspace of OAM $o_{|l|}$. A second qubit can then be added in the $\pi$ space, once that the $\pi \rightarrow o_{|l|}$ transfer has been completed $[4,10]$. The probabilistic nature of the demonstrated implementation is due to elements in the setup that discard half of the information encoded in different OAM subspaces $\left(o_{|l|} \rightarrow \pi\right)$ or in the polarization $\left(\pi \rightarrow o_{|l|}\right)$. However, a useful quantum information processing requires high efficiencies. Therefore, the demonstration of a lossless transferrer, ideally allowing for a qubit transfer with certainty (success probability $p=1$ ), is an im- portant goal. Schemes have been proposed in order to achieve this goal [11], but hitherto they have not been demonstrated experimentally.

In this paper we report the experimental implementation of a deterministic transferrer $\mathrm{O}_{2} \rightarrow \pi$ based on a q-plate and a polarizing Sagnac interferometer. In particular in this experiment we employ q-plates with topological charge $q=1$ with tuning controlled by an electric field, which allows to achieve a higher efficiency of the device, and motorized wave-plates, so that the transfer process is entirely automatized. Moreover, the same experimental setup can be also used for the inverse, $\pi \rightarrow O_{2}$ process, by reversing the propagation direction of light.

Let us first describe the working principle of the deterministic transferrer, considering the $o_{|l|} \rightarrow \pi$ process. Let us assume that the incoming photon is prepared in an arbitrary OAM and fixed polarization state, so that all information is encoded in the OAM:

$$
|H\rangle_{\pi}|\phi\rangle_{o_{|l|}}=|H\rangle_{\pi}(\alpha|+l\rangle+\beta|-l\rangle)_{o_{|l|}}
$$

where $H / V$ denotes the horizontal/vertical linear polarization. The state passes through a half waveplate (HWP) rotated at $\pi / 8$ which transforms the polarization in a diagonal one: $|A\rangle=\frac{|H\rangle+|V\rangle}{2}$, so that the state reads:

$$
|H\rangle_{\pi}(\alpha|+l\rangle+\beta|-l\rangle)_{o_{|l|}}+|V\rangle_{\pi}(\alpha|+l\rangle+\beta|-l\rangle)_{o_{|l|}}
$$

Hereafter the indices $\pi$ and $o_{|l|}$ are omitted for brevity. The photon is then sent into a polarizing Sagnac interferometer (PSI) with a polarizing beam-splitter (PBS) input/output port and a Dove prism (DP) in one of its arms [11,12]. Defining $\gamma$ as the angle between the base of the prism and the plane of the interferometer, the action of the DP on the counter-propagating $H / V$ linear polarization components with generic OAM $l$ is described by the following equations:

$$
\begin{aligned}
|H\rangle|l\rangle & \rightarrow e^{2 i l \gamma}|H\rangle|l\rangle, \\
|V\rangle|l\rangle & \rightarrow e^{-2 i l \gamma}|V\rangle|l\rangle
\end{aligned}
$$


where the OAM-inverting effect of the reflections can be ignored, for simplicity, as long as the total number of reflections in the setup is even. Thus the two components of state 2 in the PSI evolve as:

$\alpha|H\rangle|+l\rangle+i \beta|H\rangle|-l\rangle \rightarrow \alpha e^{2 i \gamma l}|H\rangle|+l\rangle+i \beta e^{-2 i \gamma l}|H\rangle|-l\rangle$, $\alpha|V\rangle|+l\rangle-i \beta|V\rangle|-l\rangle \rightarrow \alpha e^{-2 i \gamma l}|V\rangle|+l\rangle-i \beta e^{2 i \gamma l}|V\rangle|-l\rangle$. Setting $\gamma=\pi /(8 l)$ and applying these transformations to state 2 , one obtains (up to a global phase factor) the output state:

$$
\alpha|R\rangle|+l\rangle+\beta|L\rangle|-l\rangle
$$

where $L / R$ denote left/right circular polarization. By passing through a q-plate, such state is hence transformed in:

$$
(\alpha|L\rangle+\beta|R\rangle)|0\rangle_{o}=|\phi\rangle_{\pi}|0\rangle_{o}
$$

that completes the transfer. Since all the intermediate transformation steps are unitary, they are deterministic and reversible. The inverse process $\pi \rightarrow o_{|l|}$, is therefore obtained by simply inverting the light propagation through the same components. It is also interesting to note that the action of the transferrer is not limited to $\mathrm{a}+l$ and $-l$ OAM subspace, but it works with any pair $l_{1}, l_{2}$ of OAM values. By repeating the analysis above, one finds that the transfer is ensured as long as the following general condition on the DP angle is satisfied:

$$
\gamma=\frac{\pi}{4\left(l_{1}-l_{2}\right)}
$$

In this more general case, however, the final polarization state is not $R$ but depends on the values of OAM involved.

The experimental setup we used for demonstrating the deterministic $\mathrm{O}_{2} \rightarrow \pi$ transfer process can be divided in three sections: (i) generation of single photons carrying the OAM input qubit, (ii) quantum transferrer, and (iii) output state analysis (see Fig.1). In section (i) of the apparatus, an ultraviolet (UV) beam with wavelength $\lambda_{p}=397.5 \mathrm{~nm}$ pumps a $1.5 \mathrm{~mm}$ thick nonlinear crystal of $\beta$-barium borate (BBO), which generates, through the spontaneous parametric downconversion (SPDC) process, pairs of photons in spatial modes $k_{A}$ and $k_{B}$ with the same wavelength $\lambda=795 \mathrm{~nm}$ and orthogonal linear polarizations $H$ and $V$. The two photons in $k_{A}$ and $k_{B}$ are then spectrally purified by interference filters with bandwidth $\Delta \lambda=3 \mathrm{~nm}$. The photon in mode $k_{A}$ is detected and acts as a trigger of the single-photon generation. The photon in mode $k_{B}$ is delivered to the main setup via a single mode fiber, thus defining its transverse spatial mode to a pure $\mathrm{TEM}_{00}$, corresponding to OAM $l=0$. After the fiber output, two wave plates compensate the polarization rotation introduced by the fiber and a polarizing beam-splitter (PBS) projects the photon onto the state $|H\rangle_{\pi}$. A quarter waveplate (QWP) and a half-waveplate (HWP) are then used for encoding an arbitrary qubit in the polarization degree of freedom of the photon, as in Eq. (1). Finally, this polarization-encoded qubit is converted into a OAM-encoded one using the $\pi \rightarrow \mathrm{O}_{2}$ probabilistic transferrer, as described in $[10,11]$. For this step we used a q-plate with $q=1$ combined with a PBS, providing conversion into the photon state

$$
|\phi\rangle_{o_{2}}=\alpha|+2\rangle+\beta|-2\rangle
$$

with a probability $p=0.5$. This completes the input state preparation stage of our apparatus (generation box in Fig.1).

This preparation stage was used in particular for generating all states belonging to the three mutually unbiased bases spanning the bidimensional OAM subspace with $l= \pm 2\left(\mathrm{o}_{2}\right)$ : $\{|+2\rangle,|-2\rangle\},\{|h\rangle,|v\rangle\},\{|a\rangle,|d\rangle\}$, where, analogously to the polarization case, we define linear superpositions of $|+2\rangle$ and $|-2\rangle$ as $|h\rangle=\frac{1}{\sqrt{2}}(|+2\rangle+|-2\rangle),|v\rangle=\frac{1}{i \sqrt{2}}(|+2\rangle-|-2\rangle)$ and $|a\rangle=\frac{1}{\sqrt{2}}(|h\rangle+|v\rangle),|d\rangle=\frac{1}{\sqrt{2}}(|h\rangle-|v\rangle)$.

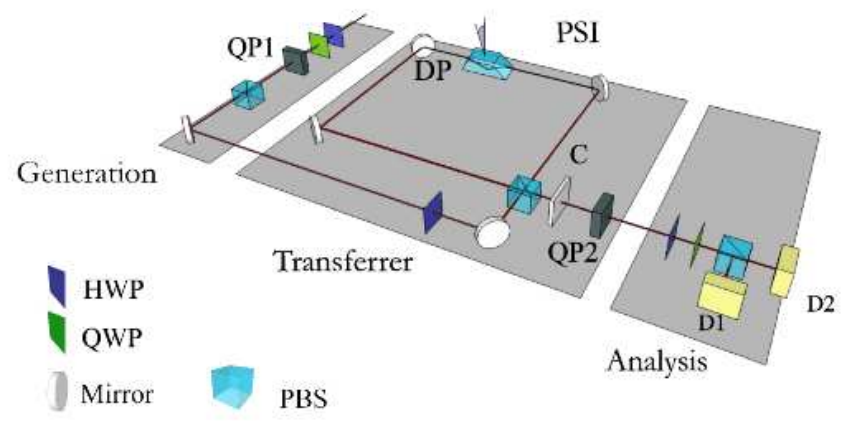

Fig. 1. Experimental setup adopted for the implementation of the deterministic quantum transferrer $O_{2} \rightarrow \pi$. The input photon, coming from the left, is prepared by a probabilistic transferrer $\left(\pi \rightarrow \mathrm{O}_{2}\right)$ (first two waveplates, QP1 and PBS) into an arbitrary $\mathrm{O}_{2}$ state with polarization $H$. After this generation stage, the PSI and the QP2 realize the deterministic transferrer $\left(\mathrm{O}_{2} \rightarrow \pi\right)$. The outgoing polarization state is analyzed in the last part of the setup (waveplates, PBS, detectors D1 and D2). $\mathrm{C}$ is a phase compensation stage to correct all the unwanted phase shifts introduced by the setup. All q-plates are electrically tuned.

For the layout of the quantum transferrer setup, i.e. section (ii) of the apparatus, we refer to Fig. 1. The input qubit $|\phi\rangle_{o_{2}}=\alpha|+2\rangle+\beta|-2\rangle$ prepared in the previous section is now passed through a half wave plate in order to set the polarization to the diagonal state $|A\rangle$ and then injected in the PSI. The first PBS of the Sagnac interferometer splits the two polarizations in two opposite directions within the PSI, both passing through a DP rotated at angle $\gamma=\frac{\pi}{16}$. The state is then sent through a q-plate (QP2), which transforms the input state to $\alpha|L\rangle+\beta|R\rangle=|\varphi\rangle_{\pi}$ with $l=0$ (mainly TEM $_{00}$ mode). Thus the information initially encoded in the orbital angular momentum has been transferred to the polarization degree of freedom.

We note that both q-plates employed in this experiment are electrically tunable. In this device, the q-plate birefringence phase retardation $\delta$ is controlled by an external electric field which changes the orientation of the liquid crystal molecular director. This allows a more convenient control of the q-plate and a faster time response as compared to the thermally-tuned q-plate $[13,14]$. By varying the applied voltage (with a sinusoidal signal) above the Fréedericksz threshold, the phase $\delta$ varies continuously between 0 and $\pi$ (or more, depending on the cell thickness). This in turn leads to a varying con- 
version efficiency of the q-plate, related to $\delta$ by a sinusoidal relation [11]. When $\delta=\pi$ the q-plate acts as a half wave plate on the polarization, which corresponds to the maximum value of conversion efficiency (tuned q-plate). In Fig.2-a we report as an example the characteristic curve of QP1, where it is shown that the optimal conversion efficiency is found to be around $4.5 \mathrm{~V}$.

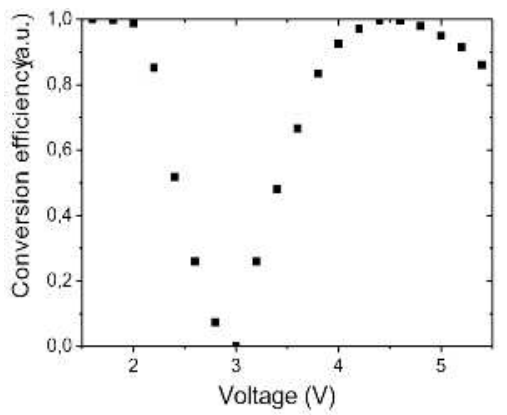

(a)

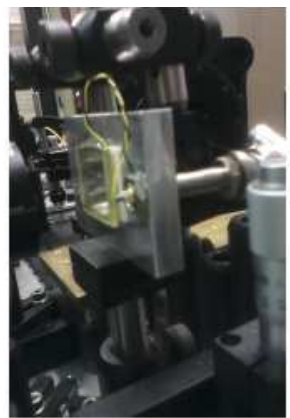

(b)
Fig. 2. (a) Conversion efficiency of the q-plate QP1 as a function of the applied voltage. Above the threshold voltage (around $2.2 \mathrm{~V}$ ), the conversion efficiency of the q-plate can be adjusted, achieving its maximum around $4.5 \mathrm{~V}$. (b) Photo of the q-plate.

After passing through the PSI and the second q-plate, all the information encoded in the input qubit is transferred to the polarization, and thus can be analyzed by a standard analysis setup made of waveplates and a polarizing beam splitter, which form the final section (iii) of our apparatus. The transmitted and reflected photons from the PBS are coupled to single mode fibers and detected by single photon counter modules $D_{1}$ and $D_{2}$. For full qubit tomography, the output of the deterministic transferrer has been analyzed in the three polarization bases $\{|R\rangle,|L\rangle\},\{|H\rangle,|V\rangle\},\{|A\rangle,|D\rangle\}$, recording the coincidence counts between detectors $\left[D_{1}, D_{T}\right]$ and $\left[D_{2}, D_{T}\right]$. The overlap between the input OAM qubit and the polarization output one after the transferrer has been estimated through the fidelity parameter $F=\frac{C_{\max }}{C_{\max }+C_{\min }}$. All results are summarized in Table 1 , showing that very good values of transfer fidelity are obtained.

Although ideally the implemented setup has success prob-

\begin{tabular}{|c|c|}
\hline \hline State & Fidelity \\
\hline \hline$|+2\rangle$ & $(0.994 \pm 0.003)$ \\
$|-2\rangle$ & $(0.992 \pm 0.003)$ \\
$|h\rangle$ & $(0.982 \pm 0.005)$ \\
$|v\rangle$ & $(0.944 \pm 0.008)$ \\
$|a\rangle$ & $(0.992 \pm 0.003)$ \\
$|d\rangle$ & $(0.980 \pm 0.005)$ \\
\hline Average value & $(0.980 \pm 0.002)$ \\
\hline
\end{tabular}

Table 1. Experimental fidelity of the qubit transfer.

ability $p=1$, the actual value is limited by standard optical losses in the optical components (mainly reflections, plus a little scattering and absorption) and by the final singlemode fiber coupling step that we used for experimental convenience and for mode purification, thus leading to an overall efficiency of 0.324 . This value is three times larger than the one achieved with the probabilistic device [11]. The obtained improvement is attributed to the adoption of the deterministic scheme based on the Sagnac interferometer, to more efficient q-plates and to a better mode conversion exemplified by a higher single-mode coupling efficiency (compared to the one measured with previous q-plates) equal to 0.30 . As further improvements the reflection losses could be reduced by adopting anti-reflection coating (in particular the q-plates are currently uncoated). Finally we note the single-mode fiber coupling (currently 0.5), although convenient for further processing of the output photons, is not a strictly required step.

In summary, we have reported the experimental implementation of a device that can transfer a qubit between the orbital angular momentum and polarization degrees of freedom of single photons. The ideal efficiency of the demonstrated device is one, so that the device is theoretically deterministic. The scheme is based on the combination of a q-plate with a Sagnac interferometer containing a Dove prism. While the reported data refer to the OAM to spin qubit transfer only, the same scheme can be used also to implement the inverse process by simply reversing the direction of light propagation in the same setup.

This work was supported by the Future and Emerging Technologies (FET) programme within the Seventh Framework Programme for Research of the European Commission, under FET-Open Grant No. 255914, PHORBITECH. CHM acknowledges the financial support from CNPq (Brazil).

\section{References}

1. L. Allen, M. W. Beijersbergen, R. J. C. Spreeuw, and J. P. Woerdman, Phys. Rev. A 45, 8185 (1992).

2. V. Bazhenov, M. Soskin, and M. Vasnetsov, J. Mod. Opt. 39, 985 (1992).

3. G. Molina-Terriza, J. P. Torres, and L. Torner, Nature Phys. 3, 305 (2007).

4. E. Nagali, L. Sansoni, L. Marrucci, E. Santamato, and F. Sciarrino, Phys. Rev. A 81, 052317 (2010).

5. E. Nagali et al., Phys. Rev. Lett. 105, 073602 (2010).

6. N. Heckenberg, R. McDuff, C. Smith, and A. White, Opt. Lett 17, 221 (1992).

7. M. Beijersbergen, L. Allen, H. van der Veen, and J. Woerdman, Opt. Commun. 96, 123 (1993).

8. M. Beijersbergen, R. Coerwinkel, M. Kristensen, and J. Woerdman, Opt. Commun. 112, 321 (1994).

9. L. Marrucci, C. Manzo, and D. Paparo, Phys. Rev. Lett. 96, 163905 (2006).

10. E. Nagali et al., Phys. Rev. Lett. 103, 013601 (2009).

11. E. Nagali et al., Opt. Express 17, 18745 (2009).

12. S. Slussarenko, V. D’Ambrosio, B. Piccirillo, L. Marrucci, and E. Santamato, Opt. Express 18, 27205 (2010).

13. E. Karimi, B. Piccirillo, E. Nagali, L. Marrucci, and E. Santamato, Appl. Phys. Lett. 94, 231124 (2009).

14. B. Piccirillo, V. D'Ambrosio, S. Slussarenko, L. Marrucci, and E. Santamato, Appl. Phys. Lett. 97, 241104 (2010). 